\section{Stroke Imaging: Diffusion, Perfusion, but No More Confusion!}

\author{
M. Wintermark, P. Sanelli, and C.C. Meltzer
}

A fter attending numerous sessions dedicated to stroke imaging at national conferences, one cannot help but leave confused with unanswered questions because the presentations consist of diametrically opposed positions regarding the imaging of patients with acute stroke. For example, some presenters advocate for the use of advanced imaging techniques, while others maintain that noncontrast CT alone is indicated to guide treatment decisions. Perfusion imaging is praised by some yet criticized by others regarding its added value in the management and treatment of patients with acute stroke. Additionally, there is no consensus regarding the preferred use of CT or MR imaging in the acute stroke setting. Therefore, it has become challenging to standardize and optimize the imaging evaluation of patients with suspected acute stroke across sites.

A wide variety of imaging techniques has become available to assess vascular lesions and brain tissue status in patients with acute stroke. In addition to scientific evidence of effectiveness, important variables that influence imaging choices include constraints of time, cost, access to imaging modalities, preferences of treating physicians, availability of expertise, and availability of endovascular therapy.

In the article entitled, "Imaging Recommendations for Acute Stroke and Transient Ischemic Attack Patients: A Joint Statement by the American Society of Neuroradiology, the American College of Radiology, and the Society of NeuroInterventional Surgery," ${ }^{1}$ we are proposing a simple, pragmatic approach that will allow the reader to develop an optimal imaging algorithm for patients with stroke at their institution.

The key elements of this consensus article can be summarized as follows:

- The primary goal of imaging patients with acute stroke symptoms is to distinguish between hemorrhagic and ischemic stroke. In patients with ischemic stroke, secondary goals of imaging before initiating revascularization interventions with intravenous thrombolysis or endovascular therapies include identification of the location and extent of intravascular clot and the presence and extent of infarct (irreversibly damaged tissue) and ischemic penumbra (hypoperfused tissue at risk for infarction).

- Early identification of the stroke etiology or mechanism (carotid atherosclerotic disease or other treatable causes) is critical to treatment decisions and long-term management.

- There is strong evidence supporting the use of IV tissue plasminogen activator as a recanalization therapy to improve clinical outcomes during the 0 - to 3 -hour time window and during the 3 to 4.5 -hour time window following symptom onset. This benefit is despite an increased risk of symptomatic intracranial hemor-

http://dx.doi.org/10.3174/ajnr.A3691 rhage after infusion. The timely use of imaging of the brain to exclude hemorrhage in patients with the clinical diagnosis of stroke and before initiating IV thrombolytic therapy is supported by strong evidence and FDA guidelines. In patients with acute stroke who are candidates for IV thrombolysis (0- to 4.5-hour time window), either noncontrast CT or MR imaging of the brain is recommended to exclude intracranial hemorrhage and determine the extent of ischemic changes. Most important, imaging in patients who are potential candidates for IV thrombolysis should not delay administration of IV thrombolysis, because "time is brain."

- There is limited evidence supporting the use of intra-arterial thrombolytic agents up to 6 hours after symptom onset. Also, the evidence supporting improved clinical outcomes with first-generation mechanical embolectomy devices up to 8 hours following symptom onset, compared with standard medical care, has been challenged by the results of the Mechanical Retrieval and Recanalization of Stroke Clots Using Embolectomy (MR RESCUE), ${ }^{2}$ Interventional Management of Stroke (IMS III), ${ }^{3}$ and Intra-arterial Versus Systemic Thrombolysis for Acute Ischemic Stroke $\left(\right.$ SYNTHESIS EXP) ${ }^{4}$ trials. Although the next generation of mechanical embolectomy devices (stent-retrievers) has received FDA approval, their clinical efficacy has not yet been established. In patients with acute stroke who are candidates for endovascular therapy, vascular imaging (CTA, MRA, conventional angiography) is strongly recommended during the initial imaging evaluation. Perfusion imaging may be considered to assess the target tissue "at risk" for reperfusion therapy. However, the accuracy and usefulness of perfusion imaging to identify and differentiate viable tissue have not been well-established.

- In patients with acute stroke, vascular imaging of the head and neck should be performed to evaluate the mechanism of stroke and assess the risk of future stroke.

These recommendations are detailed in our article. The strength of the available evidence supporting various imaging options is presented as well as considerations of available resources. It is our intention that this review and its recommendations will provide a foundation for optimizing the value of imaging in patients with acute stroke.

\section{REFERENCES}

1. Wintermark M, Sanelli P, Albers GW, et al. Imaging recommendations for acute stroke and transient ischemic attack patients: a joint statement by the American Society of Neuroradiology, the American College of Radiology, and the Society of NeuroInterventional Surgery. AJNR Am J Neuroradiol 2013;34:E117-E27

2. Kidwell CS, Jahan R, Gornbein J, et al. A trial of imaging selection and endovascular treatment for ischemic stroke. $N$ Engl J Med 2013;368:914-23

3. Broderick JP, Palesch YY, Demchuk AM, et al. Endovascular therapy after intravenous T-PA versus T-PA alone for stroke. $N$ Engl J Med 2013;368:893-903

4. Ciccone A, Valvassori L, Nichelatti M, et al, for the SYNTHESIS Expansion Investigators. Endovascular treatment for acute ischemic stroke. N Engl J Med 2013;368:904-13 